\title{
A new structural model of the acid-labile subunit: pathogenetic mechanisms of short stature-causing mutations
}

\author{
Alessia David ${ }^{*}$ Lawrence A Kelley* and Michael J E Sternberg \\ Centre for Integrative Systems Biology and Bioinformatics, Division of Molecular Biosciences, Department of Life Sciences, Imperial College London, London SW7 2AZ, UK \\ (Correspondence should be addressed to A David; Email: alessia.david09@imperial.ac.uk) \\ *(A David and L A Kelley equally contributed to this work)
}

\begin{abstract}
The acid-labile subunit (ALS) is the main regulator of IGF1 and IGF2 bioavailability. ALS deficiency caused by mutations in the ALS (IGFALS) gene often results in mild short stature in adulthood. Little is known about the ALS structure-function relationship. A structural model built in 1999 suggested a doughnut shape, which has never been observed in the leucinerich repeat (LRR) superfamily, to which ALS belongs. In this study, we built a new ALS structural model, analysed its glycosylation and charge distribution and studied mechanisms by which missense mutations affect protein structure. We used three structure prediction servers and integrated their results with information derived from ALS experimental studies. The ALS model was built at high confidence using Toll-like receptor protein templates and resembled a horseshoe with an extensively negatively charged concave surface. Enrichment in prolines and disulphide bonds was found at the ALS N- and C-termini. Moreover, seven $\mathrm{N}$-glycosylation sites were identified and mapped. ALS mutations were predicted to affect protein structure by causing loss of hydrophobic interactions (p.Leu134GIn), alteration of the amino acid backbone (p.Leu241Pro, p.Leu172Phe and p.Leu244Phe), loss of disulphide bridges (p.Cys60Ser and p.Cys540Arg), change in structural constrains (p.Pro73Leu), creation of novel glycosylation sites (p.Asp440Asn) or alteration of LRRs (p.Asn276Ser). In conclusion, our ALS structural model was identified as a highly confident prediction by three independent methods and disagrees with the previously published ALS model. The new model allowed us to analyse the ALS core and its caps and to interpret the potential structural effects of ALS mutations.
\end{abstract}

Journal of Molecular Endocrinology (2012) 49, 213-220

\section{Introduction}

The acid-labile subunit (ALS) is the main regulator of IGF1 and IGF2 bioavailability. It is a GH-dependent protein of $85 \mathrm{kDa}$ encoded by the IGFALS (Baxter 1990). ALS is present almost exclusively in the serum, where it circulates bound into a high-molecular weight ternary complex of $150 \mathrm{kDa}$. This is formed by a molecule of ALS, a molecule of IGF1 or -2 and a molecule of IGF binding protein (IGFBP) -3 or -5 . Formation of the ternary complex limits IGFs' passage through the endothelial barrier, restricting its presence to the blood circulation. This results in a reduced IGF availability to bind to its cell surface receptor (IGF1R) and to exert its mitogenic and metabolic effects (Boisclair et al. 2001, David et al. 2011).

The first homozygous mutation of IGFALS was reported in 2004 (Domene et al. 2004). The patient presented with extreme deficiencies in circulating IGF1, IGFBP3 and ALS, as well as relatively mild growth failure. The same phenotype has been observed in another 21 ALS-deficient patients, in whom an $\sim 2$ s.D. height loss was present. Interestingly, postpubertal catch-up can occur and a normal height has been observed in some ALS-deficient patients in adulthood (Domene et al. 2007a, 2009). Single heterozygous ALS mutations also appear to cause minor height loss of $\sim 1$ s.D. compared with wild-type patients (Fofanova-Gambetti et al. 2010), raising the question of whether they can be included among the causes of idiopathic short stature (Domene et al. 2004).

Little is known about the ALS structure-function relationship and the interaction between ALS and IGFBP/IGFs, although a charge interaction is hypothesised. ALS enzymatic desialisation results in a mild reduction of ALS affinity for IGFBP3 (Janosi et al. $1999 a$ ), thus suggesting the presence of additional negatively charged elements.

Experimental determination of the ALS structure would greatly aid our understanding of ternary complex formation, but this is yet to be achieved due to the difficulties in protein crystallisation. An ALS model was built in 1999 using the porcine ribonuclease inhibitor (p-RI) structure. It predicted a doughnut shape for the ALS protein (Janosi et al. 1999b), which has never been observed in the leucine-rich repeat (LRR) superfamily to which ALS belongs. Since then, there have been major advances in protein structure 
prediction and remote homology detection, as well as a far greater number of known protein structures, which allow us to confidently remodel the ALS.

In this study, we built a new structural model of the ALS protein using state-of-the-art bioinformatics tools integrated with information derived from protein databanks and ALS experimental studies. We analysed this model with respect to its charge distribution and explored potential mechanisms by which ALS missense mutations may affect the protein structure.

\section{Materials and methods}

\section{Homology modelling of ALS}

The ALS amino acid sequence was retrieved from UNIPROT database (id P35858). Homology modelling was used to determine the ALS 3D structure using three high-performing protein structure prediction servers: Phyre2 (Kelley \& Sternberg 2009, Jefferys et al. 2010), HHpred (Soding et al. 2005) and I-Tasser (Roy et al. 2010). All these methods construct protein models by detection of homology between a submitted protein sequence and proteins of known structure. They can routinely and confidently detect remote homologous relationships even when sequence identity is $<30 \%$ and have been shown to be amongst the best systems of their kind in blind trials of structure prediction (Moult et al. 2011). Both HHpred and Phyre2 use hidden Markov model matching to detect remote homology. In addition, the in-house-developed Phyre2 performs multiple template modelling and ab initio modelling of regions for which no structural template is available, using the recently in-house-developed Poing folding simulation system (Jefferys et al. 2010). I-TASSER builds structure models based on the assembly of predicted homologous substructures, using multiple-threading approaches; insight on protein function is derived by matching the predicted models with protein function databases.

The models generated by the three servers were compared by superposing them using the MaxSub procedure (Siew et al. 2000). This method attempts to find the largest subset of equivalenced residues that can be superposed within $3.5 \AA$. This is the established method for comparing models in protein structure prediction. Moreover, the same method was used to assess the deviations of the Phyre2 ALS from templates after superimposition of the templates.

\section{ALS sequence and structure analysis}

The signal peptide cleavage site was calculated using SignalP3 (Bendtsen et al. 2004), the residue accessible solvent area (asa) using DSSP (Joosten et al. 2011). The following interactions involved in protein stability were considered: i) salt bridges, defined as at least one pair of atoms on oppositely charge groups within a $4.5 \AA$ distance; ii) hydrogen bonds (H-bond), defined as a donor-acceptor distance $\leq 2.5 \AA$ and an angle at the acceptor $\geq 90^{\circ}$; and iii) disulphide bridge (S-S bridge) defined as the side chains of two cysteines at a $3 \cdot 0 \AA$ distance. Pairs of cysteines at a greater distance were also considered as potentially forming an S-S bridge when found to be reasonably close $(\mathrm{C} \alpha-\mathrm{C} \alpha$ distance $<10 \AA$ ). We used $\mathrm{C} \alpha$ distance to allow for errors in side-chain placement and a relatively high threshold to accommodate possible deviation of the backbone from native.

$\mathrm{N}$-glycosylation sites were determined based on the consensus sequence Asn-X-Thr/Ser ( $\mathrm{X}=$ any amino acid, except proline). As surface accessibility is a requirement for $\mathrm{N}$-glycosylation to occur in vivo, a manual analysis was performed to identify and exclude predicted sites located within the protein core.

$O$-glycosylation sites were predicted using GPP (Hamby \& Hirst 2008), NetOGlyc (Hansen et al. 1998) and CKSAAP_OGlySite (Chen et al. 2008) servers. As no consensus sequence is known to date and $O$-glycosylation is dependent on the structural context and surface accessibility (Calvete \& Sanz 2008), the in silico prediction was entirely based on these servers, which use complex prediction algorithms, and no additional manual analysis was performed.

The ALS electrostatic potential was calculated using PBEQ program (Jo et al. 2008), which computes the protein electrostatic potential by solving the Poisson-Boltzmann equation.

\section{Structural analysis of ALS missense mutations}

The structural impact of in-frame insertions and missense mutations identified in ALS-deficient patients was assessed by analysis of the occurrence of the following structurally important changes: i) loss of salt bridges, ii) loss of H-bond, iii) breakage of S-S bridge, iv) loss or introduction of $N$ or $O$-glycosylation sites, v) reduced hydrophobic interaction, i.e. loss of burial of $50 \AA^{2}$ or more of a non-polar area on folding, vi) introduction of a steric clash preventing formation of the native state, defined as a distance $<2.5 \AA$ between the closest atoms of two residues (side-chain position predicted using the backbone-dependent rotamer library in Pymol), and vii) alteration of a backbone constraint that can occur when proline replaces any another amino acid or when an amino acid replaces glycine.

\section{ALS structure visualisation}

Protein structures were visualised using the Pymol visualisation program (http://www.pymol.org/) and PDB structural files. 


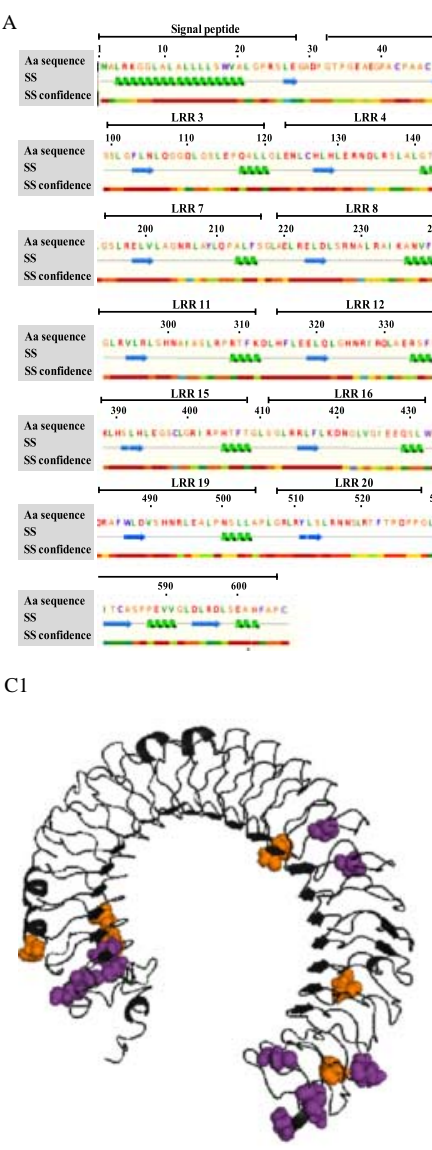

B
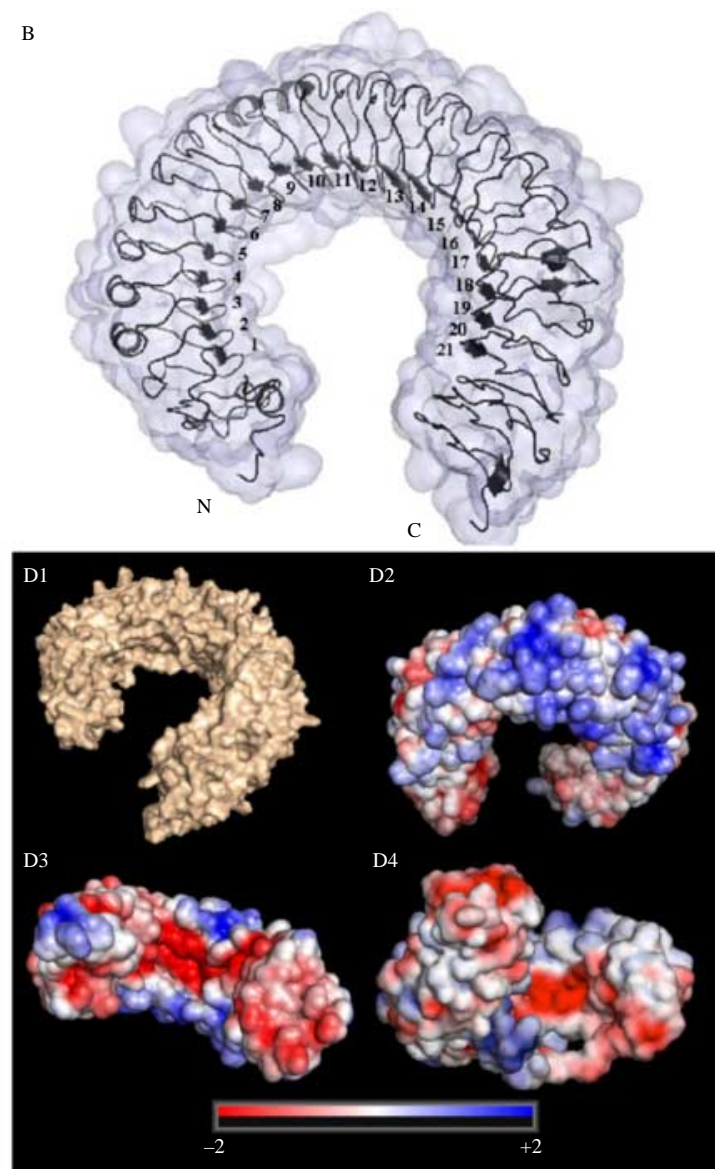

Figure 1 ALS sequence and structure analysis. (A) ALS secondary structure superimposed to its amino acid sequence. $\alpha$-Helices are presented in green, $\beta$-strands in blue and coils with a black line. The confidence in the prediction (SS confidence) is also shown. The position of the signal peptide and the LRRs is also presented. (B) ALS ribbon and surface representation of the ALS protein. LRRs are numbered from $N$ to $C$ terminus. (C) (C1) location of asparagine residues (orange spheres) representing $N$-glycosylation sites and cysteines (purple spheres) forming S-S bonds. (C2) location of proline residues (blue spheres). (D) Graphic views of the ALS solvent-accessible surface with electrostatic potential. (D1) ALS structure, (D2) ALS convex surface, (D3) and (D4) ALS inner concave surface. The electrostatic potential is visualised using a continuous colour scale ranging from negative (corresponding to $-2 \mathrm{kcal} /(\mathrm{mol} \cdot \mathrm{e})$, represented in red) to positive (corresponding to $+2 \mathrm{kcal} /(\mathrm{mol} \cdot \mathrm{e})$, represented in blue). White corresponds to $0 \mathrm{kcal} /(\mathrm{mol} \cdot \mathrm{e})$. Protein structures were visualised using Pymol. Full colour version of this figure available via http://dx.doi.org/10.1530/ JME-12-0086.

\section{Results}

\section{ALS structure model}

All three servers generated highly similar ALS models based on Toll-like receptor templates, as shown by the plots of pairwise distances between equivalent $\mathrm{C} \alpha$ residues for each superposition (available in the ALS model-dedicated website http:/ /www.sbg.bio.ic.ac. uk/phyre2/als/, which provides all supplementary material to this study). The three servers used the same or highly homologous templates. In particular, Toll-like receptors 3 and 4 were used by all servers (see Supplementary material provided in the ALS model-dedicated website, in which the sequence alignment between the ALS protein and the templates is also provided). Both Phyre2 and HHpred returned confidence estimates $>99 \%$, reflecting the confidence in the homology between ALS and the Toll-like receptor family, while I-Tasser returned a Z-score of $6 \cdot 8$ (a score $>1$ indicates good alignment).

All subsequent analyses were performed using the final model provided by our in-house-developed server Phyre2. This model is based on the following six pdb structures: 3fxi (Park et al. 2009), 2a0z (Bell et al. 2005), 2z64 (Kim et al. 2007), 3j0a (Zhou et al. 2012), 2z63 (Kim et al. 2007) and 1ziw (Choe et al. 2005). A plot of the deviations of the ALS model from the six templates after superimposition of the templates is available at http://www.sbg.bio.ic.ac.uk/phyre2/als/. 
The ALS model coordinates are available for download from the web page under 'Phyre2 results for ALS in intensive model'. The first 27 residues are predicted to form the signal peptide, with the mature protein starting at alanine 28 (Fig. 1A). The ALS model resembles a horseshoe and is characterised by 21 LRR $\beta-\alpha$ structural units (Fig. 1B).

\section{ALS structure analysis: $\mathbf{N}$ - and $\mathbf{O}$-glycosylation and electrostatic potential}

Six S-S bridges were identified (Fig. 1C1). They are clustered mainly towards the N-terminal (Cys41-Cys47 and Cys45-Cys60; LRR1) and C-terminal (Cys540Cys583, Cys542-Cys605 and Cys566-Cys571; LRR21) caps. One S-S bridge is located in the central region (Cys373-Cys397, LRR14 and -15 respectively).

Seven potential $N$-glycosylation sites (residues 64,85 , $96,368,515,554$ and 580) were identified. Six of these are located towards the $\mathrm{N}$ - and C-termini, whereas one site is located in the central region of the ALS (LRR14) and points towards the inner concave cavity of the protein. A manual analysis of these predicted $\mathrm{N}$-glycosylation sites was performed to exclude sites not accessible for glycosylation in vivo because of their position within the core of the protein. All sites resulted solvent accessible. When we assessed the presence of $O$-glycosylation sites, 14 such sites were predicted by GPP, eight of which were located towards the $\mathrm{N}$ - and C-termini. Moreover, 12 out of 14 sites (Thr66, Ser87, Ser215, Ser304, Thr309, Ser333, Ser517, Thr522, Ser556, Thr582, Ser585 and Ser598) were located on the outer convex surface and two (Ser150 and Thr442) on the inner concave surface. CKSAAP predicted one O-glycosylation site (Thr520), but at a very low confidence (prediction score $=0 \cdot 014$, threshold $=0 \cdot 000$ ). No sites were predicted by NetOGlyc.

ALS N- and C-terminal regions are rich in proline residues (Fig. 1C2). Prolines are also present in the LRR, far from the $\beta$-strands. Visualisation of the ALS electrostatic potential showed positively charged patches on the outer surface and a predominance of negatively charged patches on the inner concave surface (Fig. 1D).

\section{Structural analysis of ALS short stature-causing mutations}

Missense and in-frame mutations identified in homozygosity or compound heterozygosity in the IGFALS of patients with short stature were retrieved from the literature (Table 1) and mapped onto the ALS structure. All defects are reported to result in low or absent levels of the ALS protein in the patients' blood circulation. When we studied the impact of these mutations on protein folding and stability, we found the following:

i) Substitution of leucine residues: p.Leu241Pro, substitution of the exposed leucine with a proline can induce a main chain distortion, which would alter the ALS structure. This is further supported by the predicted side-chain torsion angles for the Leu $(\varphi, \psi=-63,-31)$, which lie on the border of the disallowed angles for Pro. Mutations p.Leu172Phe and p.Leu244Phe may affect ALS folding due to a necessary shift in the backbone to accommodate the larger side chain of phenylalanine. Finally, in p.Leu134Gln, substitution of the hydrophobic side chain of leucine with the

Table 1 IGFALS mutations identified in patients with short stature

\begin{tabular}{|c|c|c|c|}
\hline Mutation & ALS levels (mg/l) & Height (SDS) & Reference \\
\hline \multicolumn{4}{|l|}{ Missense } \\
\hline p.Asp440Asn (h.) & Undetectable & $-2 \cdot 0$ & Hwa et al. (2006) \\
\hline p.Cys540Arg (c.h.) & Undetectable & $-2 \cdot 0,-1 \cdot 0,-0 \cdot 5$ & Domene et al. $(2007 b)$ \\
\hline p.Asn276Ser (h.) & Undetectable & $-2 \cdot 4,-3 \cdot 9$ & Heath et al. (2008) \\
\hline p.Leu134GIn (h.) & NA & $-3 \cdot 2$ & Fofanova-Gambetti et al. (2009) \\
\hline p.Cys60Ser (c.h.) & Undetectable & $-2 \cdot 1$ & Fofanova-Gambetti et al. (2009) \\
\hline p.Pro73Leu (c.h.) & NA & -2.9 & Fofanova-Gambetti et al. (2009) \\
\hline p.Leu241Pro (c.h.) & NA & -2.9 & Fofanova-Gambetti et al. (2009) \\
\hline p.Leu244Phe (c.h.) & Undetectable & $-2 \cdot 1$ & Fofanova-Gambetti et al. (2009) \\
\hline p.Leu172Phe (h.) & NA & $-2 \cdot 5$ & Bang et al. (2009) \\
\hline p.Asn276Ser (c.h.) & NA & $-2 \cdot 1$ & Gallego-Gomez et al. (2009) \\
\hline p.Leu134GIn (c.h.) & 0.3 & $-2 \cdot 8$ & David et al. (2010) \\
\hline p.Pro73Leu (h.) & Undetectable & $-3 \cdot 2$ & David et al. (2010) \\
\hline \multicolumn{4}{|l|}{ In-frame insertion } \\
\hline p.Ser195-Arg197dup (c.h.) & Undetectable & $-2 \cdot 0$ & Domene et al. (2007b) \\
\hline p.Leu437-Leu439dup (h.) & Undetectable & $-2 \cdot 8$ & Fofanova-Gambetti et al. (2009) \\
\hline
\end{tabular}

NA, not available; h., patient homozygous for the mutation; c.h., patient compound heterozygous for the mutation. 
polar-charged glutamine potentially disrupts hydrophobic interactions, with a destabilising effect on ALS structure.

ii) Loss of S-S bridges: mutations p.Cys60Ser and p.Cys540Arg, Cys60 and Cys540 participate in S-S bonds with Cys45 and Cys583 respectively. Substitution of Cys60 and Cys540 is predicted to result in the loss of structurally important disulphide bridges (Fig. 2A).

iii) Alteration of LRR: mutation p.Asn276Ser, the wild-type Asn276 forms part of the LRR consensus sequence as it forms a critical hydrogen bond as part of the 'asparagine ladder' (Fig. 2B and C), stabilising the ALS core structure. In the event of substitution of asparagine with serine, the loss of the hydrogen bond could cause a substantial alteration in the ALS tertiary structure.

iv) Change in structural constrains: mutation p.Pro73Leu, proline 73 is located at the $\mathrm{N}$-terminus and is part of the structural clusters of prolines located at the $\mathrm{N}$ - and C-termini. Proline residues reduce the degree of rotation of the amino acid backbone by fixing the $\varphi, \psi$ main chain atom angles of the protein. Substitution of proline with leucine may result in the loss of this structural constraint, thus altering the ALS amino acid backbone conformation.

v) Creation of a novel glycosylation site: mutation p.Asp440Asn, this mutation occurs in a residue located on the ALS inner concave surface and creates a consensus sequence for a novel $\mathrm{N}$-glycosylation site.

vi) Disruption of the LRR arc: mutations p.Ser195 Arg197dup and p.Leu437_Leu439dup. These mutations are caused by the duplication of nine nucleotides leading to the in-frame duplication of three amino acids. The LRR units of ALS and of the other members of the LRR family form a characteristic arc or horseshoe. The LRR arc is characterised by three geometrical parameters that are derived from the solved protein structure: the tilt angle of the parallel $\beta$-strand direction turn $(\theta)$, the mean rotation angle about the central axis relating one $\beta$-strand to the next $(\varphi)$ and the inner radius of the arc $(\mathrm{R})$ (Matsushima et al. 2005). Duplication Leu 437_Leu439 alters the formation of the $\beta$-strand motif in LRR17, as residues 438 and 439 contribute to the formation of the highly conserved $\beta$-strand of the LRR (Enkhbayar et al. 2004). Ultimately, alteration of the $\beta$-strand could result in the alteration of one or more of the three geometrical parameters of the LRR arc. A similar drastic structural change in the LRR arc can be predicted for the p.Ser195_Arg197dup. This duplication involves the $\beta$-turn, which
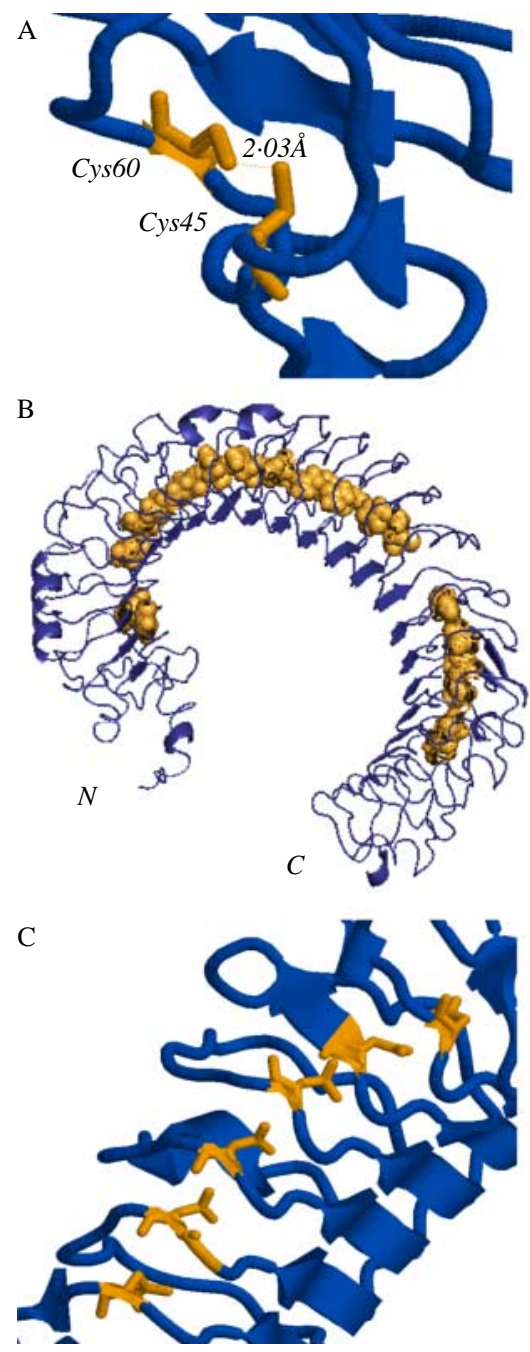

Figure 2 (A) Cartoon representation of disulphide bond between Cys45 and Cys60. Cysteine residues are presented as orange sticks. The disulphide bond is presented with a dashed line. The distance between the two cysteines is also presented. (B) Cartoon representation of the ALS asparagine (Asn) ladder. Asn residues forming the asparagine ladder are represented as yellow spheres. (C) Detailed view of the asparagine ladder. Asn residues are presented as orange sticks. Full colour version of this figure available via http://dx.doi.org/10.1530/JME-12-0086.

connects the $\beta$-strand on the concave surface with the structural motifs on the convex face and is also responsible for the 180-degree bend of the ALS backbone.

\section{Discussion}

We propose a new structural model of ALS based on results from three state-of-the-art protein structure prediction servers. This new model disagrees with the 
previously published model in 1999 (Janosi et al. 1999b) in a number of respects. The previous model was based on the p-RI, which contains LRRs composed of 28-30 residues per repeat. The authors acknowledged that this did not match the 22-27 residues per repeat present in ALS and in many other LRR-containing proteins. In addition, the earlier model lacked the $\mathrm{N}$ - and C-terminal regions, providing no accurate information on ALS caps. Finally, the authors suggested an extension of the known RI structure from a horseshoe to a doughnut to accommodate the missing residues, although no such structure has been seen in LRR proteins to date.

As a result of improvements in protein structure prediction coupled with increases in the protein structural database, we were able to confidently build a model for more than $90 \%$ of ALS residues. This new model is based on the Toll-like receptor family and is consistent with the known length of LRR in ALS. The $\mathrm{N}$ - and C-termini are enriched in proline residues and contain several potential S-S bonds, whose function is likely to be the capping of the LRR hydrophobic core. Shielding of the LRR core by N and C caps is a common feature in LRR proteins and, similar to what is observed in ALS, the $\mathrm{N}$ and C caps of extracellular LRR proteins often contain clusters of cysteines, which form disulphide bridges (Buchanan \& Gay 1996, Matsushima et $a l$. 2005). Three of the putative S-S bridges identified by our analysis are conserved features in the templates used for ALS model prediction. In particular, the N-terminal ALS C45-C60 S-S bridge $\left(\mathrm{Cx}_{14} \mathrm{C}\right.$ motif, where $\mathrm{x}$ can be any amino acid) corresponds to the $\mathrm{Cx}_{8} \mathrm{C}$ motif in the Toll-like receptor 3 (TLR3) and to the $\mathrm{Cx}_{10} \mathrm{C}$ motif in TLR4, and ALS C-terminal S-S bridges C540-C583 and C542-C605 ( $\mathrm{CxCx}_{40} \mathrm{Cx}_{21} \mathrm{C}$ motif) correspond to the two S-S bridges present in the TLR4 $\left(\mathrm{CxCx}_{23} \mathrm{Cx}_{17} \mathrm{C}\right.$ motif, sequence alignment between ALS and templates available in the Supplementary material). A manual analysis of the ALS structure identified three additional cysteine pairs. These pairs are spatially close, although at a distance greater than that allowed for the formation of disulphide bridges. However, given the inherent error/deviation of the model backbone from native, it is feasible that these pairs do form bridges in the native, though experimental testing is needed to confirm this. We used this novel ALS model to interpret the possible structural effects of known pathogenic mutations in ALS, to predict glycosylation states and to analyse its electrostatic surface.

Experimental determination of the molecular mechanisms by which a mutation affects protein function or stability is the gold standard but can be challenging. We mapped ALS in-frame insertions and missense mutations to the new model and suggested a molecular explanation of their deleterious effect using a computational approach alone. Nonsense and frameshift mutations have also been identified in patients with ALS deficiency. Their structural effect was not analysed, as they result in premature stop codons, which cause premature termination of mRNA translation. These transcripts are likely to be degraded by nonsense-mediated mRNA decay, thus preventing the synthesis of prematurely truncated mutant proteins.

Confirmation of our predictions could only be obtained for one mutation, p.Asp440Asn, which has been shown by previous experiments to cause the formation of a new glycosylation site (Firth $e t$ al. 2011). Creation of novel glycosylation sites can cause protein misfolding and rapid protein degradation (Freeze \& Schachter 2009) and could explain ALS deficiency in the patients' circulation (Hwa et al. 2006).

Identification of potential $\mathrm{N}$-glycosylation sites was achieved by identification of the NXS/T sequon. Although the $\mathrm{N}$-glycosidic bond always occurs at the amide of the asparagine in this well-known consensus sequence, the asparagine of this sequon is not always glycosylated. Fully glycosylated ALS has a molecular weight of 84-86 kDa. As the mass of the polypeptide backbone is $\sim 66 \mathrm{kDa}, N$-glycosylation contributes $\sim 20 \mathrm{kDa}$ to the ALS molecular weight (Janosi et al. 1999a). In vitro ALS enzymatic deglycosylation with increasing concentration of $\mathrm{N}$-glycosidase results in at least six isoforms corresponding to partially deglycosylated ALS (Janosi et al. 1999a). This in vitro evidence supports our in silico prediction of the presence of multiple $\mathrm{N}$-glycosylation sites. Conversely, in silico prediction of the ALS O-glycosylation sites was more challenging. $O$-glycosylation site prediction is error prone as it may depend on the structural context and surface accessibility (Calvete \& Sanz 2008), and no consensus sequence is known to date. In vitro studies have shown that the ALS protein is not extensively $O$-glycosylated, as enzymatic $O$-deglycosylation does not result in a change in ALS molecular weight (Janosi et al. $1999 a$ ) and this is consistent with the prediction results of the CKSAAP and NetOGlyc servers. Glycosylation sites provide negative charges to the ALS protein and it has been hypothesised that they are involved in the interaction with the positive charges on the IGF1/ IGFBP binary complex. Accurate localisation of these sites on the ALS structure can prove useful for designing mutagenesis studies. In fact, computational results alone rarely provide a definitive answer in complex cases, such as determination of $O$-glycosylation sites, and their exact number and position should be further assessed experimentally, before definitive conclusions can be drawn.

When the ALS surface electrostatic potential was calculated, we found that the concave surface was largely negatively charged with multiple patches extending from the $\mathrm{N}$ - to the $\mathrm{C}$-terminus of the protein. 
These negative patches could be responsible for the electrostatic interaction with IGFBP3 and -5 , thus fitting the hypothesis of a negative-positive interaction. The use of the concave surface for formation of biological complexes has been demonstrated for other members of the LRR family, such as the Toll-like receptor 3 (Choe et al. 2005).

The novel information on the ALS structure gathered in this study was used to attempt modelling of the ALS-IGFBP-IGF ternary complex (data not shown). Nevertheless, the lack of IGFBP3 or -5 solved structure and the still suboptimal accuracy of docking algorithms did not allow us to build a confident model of interaction.

Advances in the understanding of the ALS structurefunction relationship can help to identify the biological determinants of ternary complex formation and ultimately lead to the development of new drugs for the treatment of disorders, ranging from short stature to neoplasias. Although in silico observations alone rarely provide a definitive proof of structure-function relationship, they can provide invaluable information for designing experimental studies and assist in biological problem solving.

\section{Supplementary data}

This is linked to the online version of the paper at http://dx.doi.org/ 10.1530/JME-12-0086.

\section{Declaration of interest}

A D and L A K have nothing to declare. M J E S declares that he is a Director and Shareholder in Equinox Pharma Ltd, which uses computational methods for drug discovery and provides bioinformatics services. Equinox Pharma holds a licence for commercial use of some components of the Phyre2 software used in this study.

\section{Funding}

Dr L A K was supported by a BBSRC grant reference BB/G022569/1.

\section{References}

Bang P, Fureman AL, Nilsson AL, Boström J, Kriström B, Ekström K, Hwa V, Rosenfeld RG \& Carlsson-Skwirut C 2009 A novel missense mutation of the ALSIGF gene causing a L172F substitution in LRR6 is associated with short stature in two Swedish children homozygous or compound heterozygous for the mutation. Hormone Research 7286.

Baxter RC 1990 Circulating levels and molecular distribution of the acid-labile (alpha) subunit of the high molecular weight insulin-like growth factor-binding protein complex. Journal of Clinical Endocrinology and Metabolism 70 1347-1353. (doi:10.1210/ jcem-70-5-1347)
Bell JK, Botos I, Hall PR, Askins J, Shiloach J, Segal DM \& Davies DR 2005 The molecular structure of the Toll-like receptor 3 ligandbinding domain. PNAS 102 10976-10980. (doi:10.1073/pnas. 0505077102)

Bendtsen JD, Nielsen H, von Heijne G \& Brunak S 2004 Improved prediction of signal peptides: SignalP 3.0. Journal of Molecular Biology 340 783-795. (doi:10.1016/j.jmb.2004.05.028)

Boisclair YR, Rhoads RP, Ueki I, Wang J \& Ooi GT 2001 The acid-labile subunit (ALS) of the $150 \mathrm{kDa}$ IGF-binding protein complex: an important but forgotten component of the circulating IGF system. Journal of Endocrinology 170 63-70. (doi:10.1677/joe.0.1700063)

Buchanan SG \& Gay NJ 1996 Structural and functional diversity in the leucine-rich repeat family of proteins. Progress in Biophysics and Molecular Biology 65 1-44. (doi:10.1016/S0079-6107(96)00003-X)

Calvete JJ \& Sanz L 2008 Analysis of $O$-glycosylation. Methods in Molecular Biology 446 281-292. (doi:10.1007/978-1-60327-084-7_20)

Chen YZ, Tang YR, Sheng ZY \& Zhang Z 2008 Prediction of mucin-type $O$-glycosylation sites in mammalian proteins using the composition of k-spaced amino acid pairs. BMC Bioinformatics 9101. (doi:10.1186/1471-2105-9-101)

Choe J, Kelker MS \& Wilson IA 2005 Crystal structure of human Toll-like receptor 3 (TLR3) ectodomain. Science 309 581-585. (doi:10.1126/science.1115253)

David A, Rose SJ, Miraki-Moud F, Metherell LA, Savage MO, Clark AJ \& Camacho-Hubner C 2010 Acid-labile subunit deficiency and growth failure: description of two novel cases. Hormone Research in Paediatrics 73 328-334. (doi:10.1159/000308164)

David A, Hwa V, Metherell LA, Netchine I, Camacho-Hubner C, Clark AJ, Rosenfeld RG \& Savage MO 2011 Evidence for a continuum of genetic, phenotypic, and biochemical abnormalities in children with growth hormone insensitivity. Endocrine Reviews 32 472-497. (doi:10.1210/er.2010-0023)

Domene HM, Bengolea SV, Martinez AS, Ropelato MG, Pennisi P, Scaglia P, Heinrich JJ \& Jasper HG 2004 Deficiency of the circulating insulin-like growth factor system associated with inactivation of the acid-labile subunit gene. New England Journal of Medicine 350 570-577. (doi:10.1056/NEJMoa013100)

Domene HM, Martinez AS, Frystyk J, Bengolea SV, Ropelato MG, Scaglia PA, Chen JW, Heuck C, Wolthers OD, Heinrich JJ et al. 2007 a Normal growth spurt and final height despite low levels of all forms of circulating insulin-like growth factor-I in a patient with acid-labile subunit deficiency. Hormone Research 67 243-249. (doi:10.1159/ 000098479)

Domene HM, Scaglia PA, Lteif A, Mahmud FH, Kirmani S, Frystyk J, Bedecarras P, Gutierrez M \& Jasper HG 2007b Phenotypic effects of null and haploinsufficiency of acid-labile subunit in a family with two novel IGFALS gene mutations. Journal of Clinical Endocrinology and Metabolism 92 4444-4450. (doi:10.1210/jc.2007-1152)

Domene HM, Hwa V, Argente J, Wit JM, Camacho-Hubner C, Jasper HG, Pozo J, van Duyvenvoorde HA, Yakar S, Fofanova-Gambetti OV et al. 2009 Human acid-labile subunit deficiency: clinical, endocrine and metabolic consequences. Hormone Research 72 129-141. (doi:10.1159/ 000232486)

Enkhbayar P, Kamiya M, Osaki M, Matsumoto T \& Matsushima N 2004 Structural principles of leucine-rich repeat (LRR) proteins. Proteins 54 394-403. (doi:10.1002/prot.10605)

Firth SM, Yan X \& Baxter RC 2011 D440N mutation in the acid-labile subunit of insulin-like growth factor complexes inhibits secretion and complex formation. Molecular Endocrinology 25 307-314. (doi:10.1210/me.2010-0295)

Fofanova-Gambetti OV, Hwa V, Kirsch S, Pihoker C, Chiu HK, Hogler W, Cohen LE, Jacobsen C, Derr MA \& Rosenfeld RG 2009 Three novel IGFALS gene mutations resulting in total ALS and severe circulating IGF-I/IGFBP-3 deficiency in children of different ethnic origins. Hormone Research 71 100-110. (doi:10.1159/000183899)

Fofanova-Gambetti OV, Hwa V, Wit JM, Domene HM, Argente J, Bang P, Hogler W, Kirsch S, Pihoker C, Chiu HK et al. 2010 Impact of heterozygosity for acid-labile subunit (IGFALS) gene mutations on 
stature: results from the international acid-labile subunit consortium. Jourmal of Clinical Endocrinology and Metabolism 95 4184-4191. (doi:10.1210/jc.2010-0489)

Freeze HH \& Schachter H 2009 Genetic disorders of glycosylation. Genetic disorders of glycosylation. In Essentials of Glycobiology, 2nd edn, p 42. Eds A Varki, RD Cummings, JD Esko, HH Freeze, P Stanley, CR Bertozzi, GW Hart \& ME Etzler. Cold Spring Harbor, NY: Cold Spring Harbor Laboratory Press.

Gallego-Gomez E, Sanchez del Pozo J, Cruz Rojo J, Zurita-Muñoz O, Gracia-Bouthelier R, Heath KE \& Campos-Barros A 2009 Novel compound heterozygous IGFALS mutation associated with impaired postnatal growth and low circulating IGF-I and IGFBP-3 levels. Hormone Research 72 90-91.

Hamby SE \& Hirst JD 2008 Prediction of glycosylation sites using random forests. BMC Bioinformatics 9 500. (doi:10.1186/ 1471-2105-9-500)

Hansen JE, Lund O, Tolstrup N, Gooley AA, Williams KL \& Brunak S 1998 NetOglyc: prediction of mucin type $O$-glycosylation sites based on sequence context and surface accessibility. Glycoconjugate Journal 15 115-130. (doi:10.1023/A:1006960004440)

Heath KE, Argente J, Barrios V, Pozo J, Diaz-Gonzalez F, Martos-Moreno GA, Caimari M, Gracia R \& Campos-Barros A 2008 Primary acid-labile subunit deficiency due to recessive IGFALS mutations results in postnatal growth deficit associated with low circulating insulin growth factor (IGF)-I, IGF binding protein-3 levels, and hyperinsulinemia. Journal of Clinical Endocrinology and Metabolism 93 1616-1624. (doi:10.1210/jc.2007-2678)

Hwa V, Haeusler G, Pratt KL, Little BM, Frisch H, Koller D \& Rosenfeld RG 2006 Total absence of functional acid labile subunit, resulting in severe insulin-like growth factor deficiency and moderate growth failure. Journal of Clinical Endocrinology and Metabolism 91 1826-1831. (doi:10.1210/jc.2005-2842)

Janosi JB, Firth SM, Bond JJ, Baxter RC \& Delhanty PJ $1999 a$ N-linked glycosylation and sialylation of the acid-labile subunit. Role in complex formation with insulin-like growth factor (IGF)-binding protein-3 and the IGFs. Journal of Biological Chemistry 274 5292-5298. (doi:10.1074/jbc.274.9.5292)

Janosi JB, Ramsland PA, Mott MR, Firth SM, Baxter RC \& Delhanty PJ $1999 b$ The acid-labile subunit of the serum insulin-like growth factor-binding protein complexes. Structural determination by molecular modeling and electron microscopy. Journal of Biological Chemistry 274 23328-23332. (doi:10.1074/jbc.274.33.23328)

Jefferys BR, Kelley LA \& Sternberg MJ 2010 Protein folding requires crowd control in a simulated cell. Journal of Molecular Biology 397 1329-1338. (doi:10.1016/j.jmb.2010.01.074)
Jo S, Vargyas M, Vasko-Szedlar J, Roux B \& Im W 2008 PBEQ-Solver for online visualization of electrostatic potential of biomolecules. Nucleic Acids Research 36 W270-W275. (doi:10.1093/nar/gkn314)

Joosten RP, te Beek TA, Krieger E, Hekkelman ML, Hooft RW, Schneider R, Sander C \& Vriend G 2011 A series of PDB related databases for everyday needs. Nucleic Acids Research 39 D411-D419. (doi:10.1093/nar/gkq1105)

Kelley LA \& Sternberg MJ 2009 Protein structure prediction on the Web: a case study using the Phyre server. Nature Protocols 4 363-371. (doi:10.1038/nprot.2009.2)

Kim HM, Park BS, Kim JI, Kim SE, Lee J, Oh SC, Enkhbayar P, Matsushima N, Lee H, Yoo OJ et al. 2007 Crystal structure of the TLR4-MD-2 complex with bound endotoxin antagonist Eritoran. Cell 130 906-917. (doi:10.1016/j.cell.2007.08.002)

Matsushima N, Tachi N, Kuroki Y, Enkhbayar P, Osaki M, Kamiya M \& Kretsinger RH 2005 Structural analysis of leucine-rich-repeat variants in proteins associated with human diseases. Cellular and Molecular Life Sciences 62 2771-2791. (doi:10.1007/s00018005-5187-z)

Moult J, Fidelis K, Kryshtafovych A \& Tramontano A 2011 Critical assessment of methods of protein structure prediction (CASP)-Round IX. Proteins 79 (Suppl 10) 1-5. (doi:10.1002/ prot.23200)

Park BS, Song DH, Kim HM, Choi BS, Lee H \& Lee JO 2009 The structural basis of lipopolysaccharide recognition by the TLR4-MD-2 complex. Nature 458 1191-1195. (doi:10.1038/nature07830)

Roy A, Kucukural A \& Zhang Y 2010 I-TASSER: a unified platform for automated protein structure and function prediction. Nature Protocols 5 725-738. (doi:10.1038/nprot.2010.5)

Siew N, Elofsson A, Rychlewski L \& Fischer D 2000 MaxSub: an automated measure for the assessment of protein structure prediction quality. Bioinformatics 16 776-785. (doi:10.1093/bioinformatics/16.9.776)

Soding J, Biegert A \& Lupas AN 2005 The HHpred interactive server for protein homology detection and structure prediction. Nucleic Acids Research 33 W244-W248. (doi:10.1093/nar/gki408)

Zhou K, Kanai R, Lee P, Wang HW \& Modis Y 2012 Toll-like receptor 5 forms asymmetric dimers in the absence of flagellin. Journal of Structural Biology 177 402-409. (doi:10.1016/j.jsb.2011.12.002)

Received in final form 4 September 2012 Accepted 18 September 2012

Made available online as an Accepted Preprint 18 September 2012 